\title{
Capsule-Based Processing and Handling of Electron Microscopy Specimens and Grids
}

\author{
Steven L. Goodman, ${ }^{1 *}$ Kristy D. Wendt, ${ }^{1,}{ }^{2}$ Michael S. Kostrna, ${ }^{3}$ and Craig Radi ${ }^{4}$ \\ ${ }^{1}$ Microscopy Innovations LLC, 213 Air Park Road, Suite 101, Marshfield, WI 54449 \\ ${ }^{2}$ Laboratory for Optical and Computational Instrumentation, University of Wisconsin, 1675 Observatory Drive, Madison, \\ WI, 53706 \\ ${ }^{3}$ Electron Microscopy Training Program, Madison College. 1701 Wright Street, Madison, WI 53704 \\ ${ }^{4}$ Wisconsin Veterinary Diagnostic Laboratory, 445 Easterday Lane, Madison, WI 53706 \\ *steven.goodman@microscopyinnovations.com
}

\section{Introduction}

It is a testament to the brilliance of the earliest biological electron microscopists that half a century later, most cell and tissue specimens for transmission electron microscopy (TEM) are still prepared using chemical fixation, polymer embedding, microtomy, and heavy-metal staining protocols based on those developed by Palade, Porter, Fullam, Claude, and others in the 1950s-1970s [1-5]. These methods remain in use today because they provide good fixation and electron contrast, and they enable thin sectioning. To perform these protocols, biological specimens are placed into vials filled with each chemical reagent, followed by manually exchanging reagents using transfer pipettes. Similarly, grids are stained by manually moving them between reagent droplets or dishes. However, why these fluid-handling methods have remained largely unchanged in the modern electron microscopy lab is surprising because fluid handing in life science laboratories was transformed during the same decades as TEM revolutionized biology. Two leading advancements in fluid handling were the introduction of the variable-volume pipetter by Gilson [6] in 1972 and the massproduced micro-well plate (microtiter plate) invented by Cooke [7-8] and commercialized about 1966 [9].

The importance of fluid handling in electron microscopy is considerable. Typical TEM specimen preparation protocols comprise 20 or more fixative, rinse, dehydrant, and resin exchanges. Thus, the process of preparing only 5 different tissue samples entails over 200 discrete vial-filling and emptying steps. Not only is this tedious, but specimens can be damaged by contact with the container, by being accidentally aspirated into transfer pipettes, by air exposure when specimens float or stick to the vial sides, and when transfers between reagents take too long. Specimens may even be lost because many tissues are difficult to see when immersed or when they stick to the sides of vials. It is also difficult to maintain consistent timing when several specimens are being prepared, thus reducing experimental reproducibility. Although automatic tissue processers ease this tedium and equalize reagent timing, most such devices require cumbersome manual specimen transfers from porous baskets into embedding molds. More importantly, automatic processors are not able to simultaneously prepare a group of specimens with different staining and fixation protocols as commonly required in research.

Manual processing can be wasteful of reagent because common lab vessels (scintillation vials, microfuge tubes) require at least $1-2 \mathrm{ml}$ of reagent simply to keep specimens immersed, yet only 7-10 times the volume of a specimen is sufficient for each reagent step [10]. Given that biological tissue TEM specimens are typically $1 \mathrm{~mm}^{3}$, specimen volume is thus about $1 \mu \mathrm{l}$, and therefore only $7-10 \mu \mathrm{l}$ of reagent is actually required. Thus, reagent volumes consumed in vial processing easily exceed 100 times that required for sufficient reaction.

For most labs the process for staining sections on grids with heavy metals (or immuno-labels) has also not changed [11-12]. Grids are extensively handled using forceps to transfer them into and out of grid boxes, onto and between stain droplets or other staining apparatuses, back into grid boxes, and eventually onto the TEM stage. Because grids are fragile, it is challenging to not damage, drop, or lose them. Also, because individual grids are not labeled, they can easily be mixed up. It is also difficult to obtain identical timing when multiple grids are being prepared, thus reducing experimental reproducibility.

This article describes a new capsule-based processing system for preparing and handling EM specimens and TEM grids. It is based on two types of microliter-volume, purpose-built vessels called mPrep ${ }^{\mathrm{Ts}}$ capsules. Each $\mathrm{mPrep} / \mathrm{s}$ capsule (microPreparation for specimens) entraps an individual specimen, while each $\mathrm{mPrep} / \mathrm{g}$ capsule (micro-Preparation for grids) holds one or two TEM grids. Both mPrep capsule types connect to standard and widely used adjustable-volume laboratory pipettors in the same manner as pipette tips. This provides for efficient delivery of reagents directly to the specimens or grids entrapped in the capsules. Once a specimen or grid is inserted into an mPrep capsule, it may never require removal except to place grids into the microscope. This capsule system eliminates almost all specimen and grid handling, greatly reducing the potential for damage, loss, or misidentification because each capsule is readily labeled.

\section{Materials and Methods}

Several mammalian and one plant specimen were prepared and examined. Tissues from laboratory rats (Rattus norvegicus), Yucatan mini-pigs, and Dieffenbachia (dumb cane) plant tissue were prepared using $\mathrm{mPrep} / \mathrm{s}$ specimen capsule processing followed by section staining on grids using mPrep/g capsules. Sections of vampire bat brain (Desmondontidae) were stained using $\mathrm{mPrep} / \mathrm{g}$ capsule processing from specimens provided after fixation and embedding using conventional methods.

Specimen processing. Rat and pig tissues were prepared from fresh specimens provided from other research investigations. 


\section{LUMENCOR \\ CUSTOMER FOCUS}

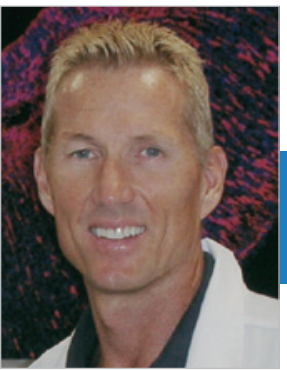

Brian D. Armstrong MICROSCOPY CORE DIRECTOR

Duarte, CA
What do you do at the Institution that potentially demands high performance, solid state, technical lighting?

Operating a Light Microscopy Core is challenging because scientists demand high performance illumination for a variety of fluorescent sample types in which sensitivity and lower detection limits vary significantly. We see samples including cells and tissues that have been labeled with dyes, antibodies and fluorescent proteins. We rely heavily on our LED systems to provide Illumination whenever we need it.

What are some of the challenges that need to be overcome to ensure optimal performance for imaging or quantitation?

In order to satisfy the...

"demands of hundreds of investigators we must provide illumination that offers sufficient intensity and stability. The Lumencor products have met our standards" ....and the ability to turn them on and off at will has made fluorescence illumination easier.

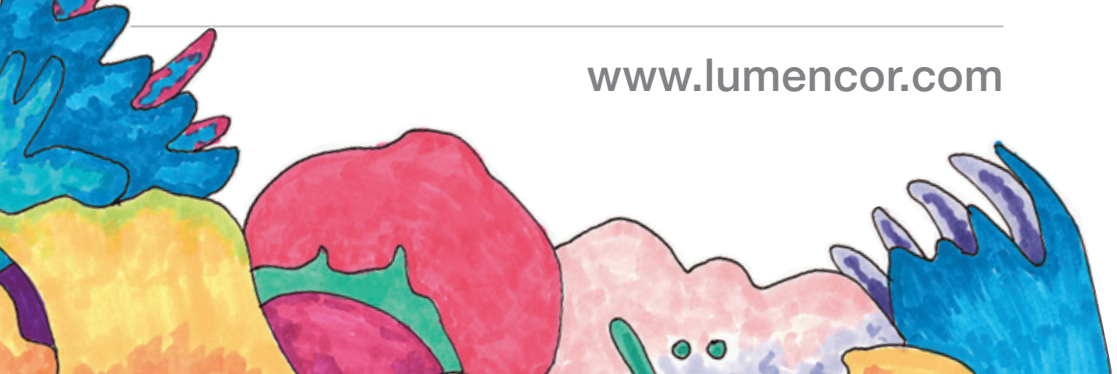

How have Lumencor's products addressed these challenges?

"Since we have changed all of our mercury sources over to the Lumencor SOLA light engines there have not been any complaints regarding brightness, stability or ease of use. We expect that the switch to LED sources will decrease our expenses with regard to utilities and hazardous waste removal."

The LED sources will also provide a safer workplace by removing the risk of explosion of mercury bulbs and from mercury exposure from broken bulbs. The switch to LED sources will also help us to further our long term goal of reducing greenhouse gases and hazardous waste.
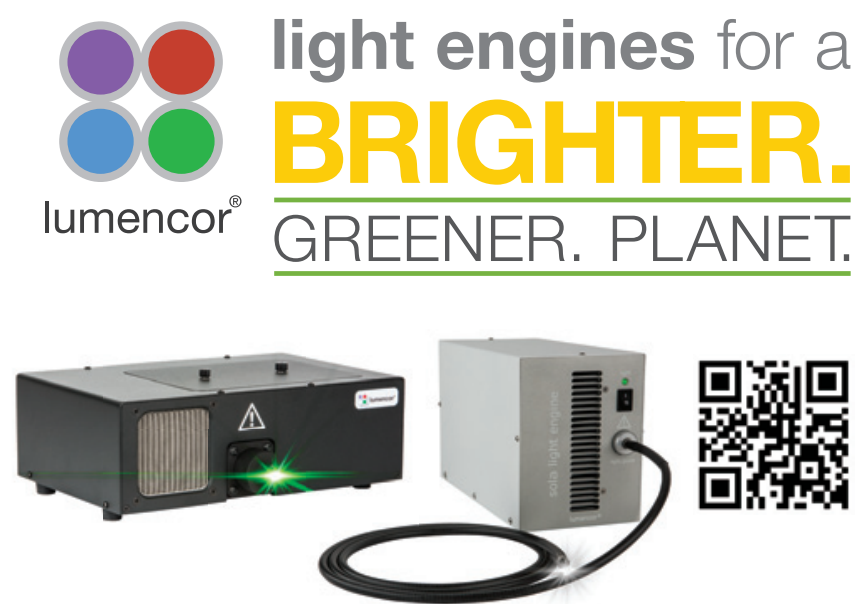
Rat kidney was perfusion fixed, excised, immersed in Karnovsky's fixative [13], and then refrigerated until subsequently it was fully processed. Pig heart and skin samples were rapidly excised after euthanasia, immediately immersed in Karnovsky's fixative, and refrigerated until subsequently processed. Tissue specimens were then cut into $1 \mathrm{~mm}^{3}$ pieces suitable for TEM preparation and entrapped into $\mathrm{mPrep} / \mathrm{s}$ capsules between the $300 \mu \mathrm{m}$ pores molded into the capsule bottom and the removable screen, which also has $300 \mu \mathrm{m}$ pores (Figure 1a). The capsules were then attached to a 12-channel Gilson Neo P12X200N Pipetman (Figure 1b) via $\mathrm{mPrep} / \mathrm{f}$ filter couplers, which prevent accidental drawing of reagents into the pipettor. To identify each specimen, adhesive labels were attached to each capsule. The labels used in this study (provided with mPrep capsules) included both a computer-readable 2D (datamatrix) barcode and humanreadable alphanumeric characters.

Tissue was processed by simultaneously aspirating $100 \mu \mathrm{l}$ of each reagent from polyethylene reservoirs (troughs) into all capsules. The pipettor was held upright with the capsules resting in the reservoir for the designated reaction time using a lab stand. The reagent sequence was:

1. Fresh Karnovsky's fixative for $1 \mathrm{hr}$ (to ensure full fixation after tissue cutting)

$2.3 \times 5$ min washes in Sorensen's sodium phosphate buffer
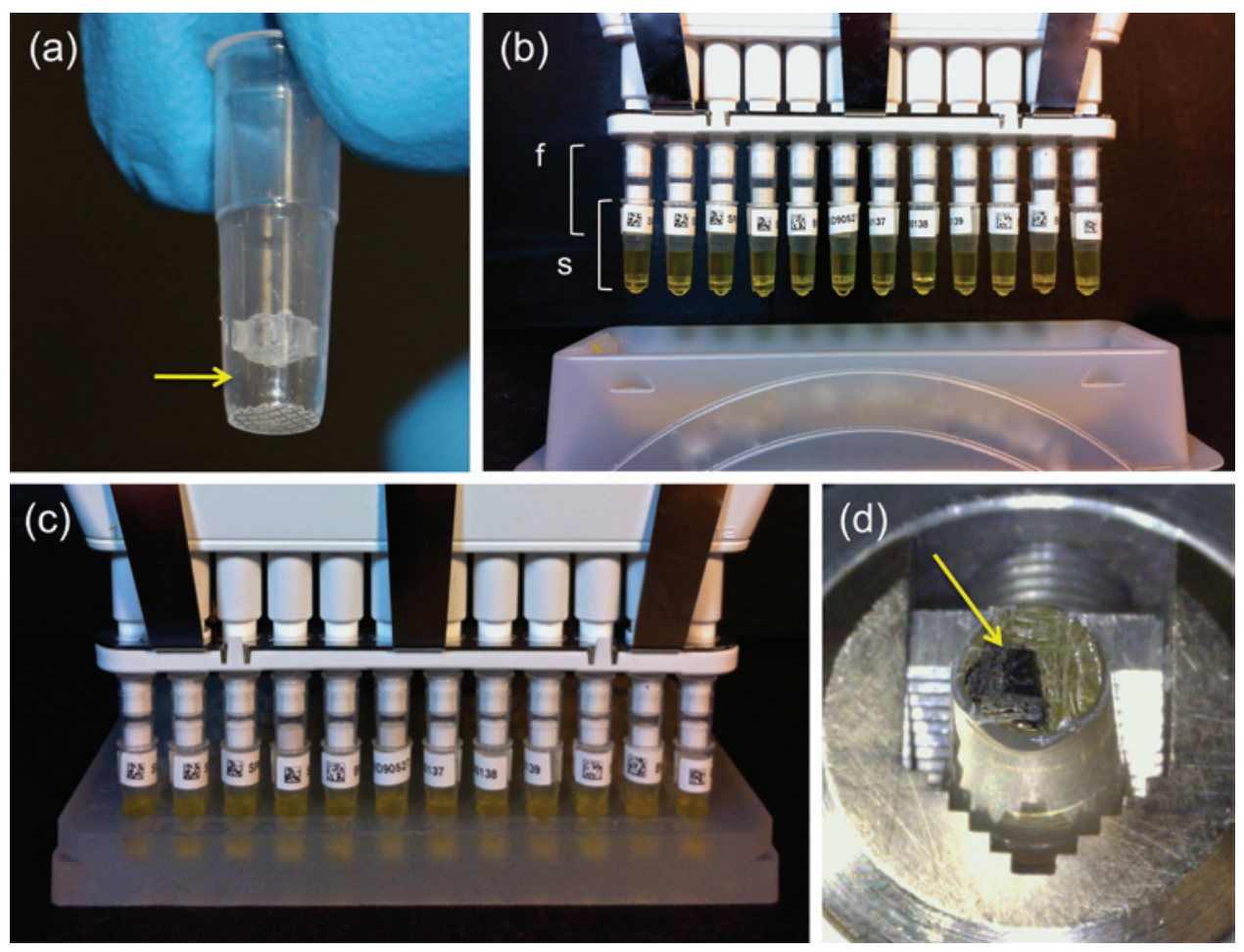

Figure 1: Tissue processing using mPrep/s capsules: (a) Empty mPrep/s capsule. The arrow indicates the location for entrapping specimens between the capsule bottom with $300 \mu \mathrm{m}$ pores, and the removable, adjustable $300 \mathrm{um}$ pore screen. For clarity no specimen is shown. The tool to insert and remove the screen is not shown. (b) Twelve labeled mPrep/s capsules [s] attached to a 12-channel Pipetman held above the reagent reservoir. Each $\mathrm{mPrep} / \mathrm{s}$ capsule contains a $\sim 1 \mathrm{~mm}^{3}$ specimen and $100 \mu \mathrm{l}$ reagent (en bloc uranyl acetate). Capsules are connected to the pipettor via an $\mathrm{mPrep} / \mathrm{f}$ filter coupler [f]. (c) Twelve $\mathrm{mPrep} / \mathrm{s}$ capsules filled with epoxy and inserted into the $\mathrm{mPrep} /$ bench just prior to ejection from the pipettor. (d) $\mathrm{mPrep} / \mathrm{s}$ capsule clamped into a microtome chuck with the capsule trimmed away to expose the epoxy-embedded tissue. The arrow shows the faced epoxy block ready for sectioning.
3. $1 \% \mathrm{OsO}_{4}(\mathrm{wt} / \mathrm{vol})$ in Sorensen's sodium phosphate buffer for $1 \mathrm{hr}$

4. $3 \times 10 \mathrm{~min}$ washes in deionized water

$5.1 \%(\mathrm{wt} / \mathrm{vol})$ aqueous uranyl acetate for $1 \mathrm{hr}$

$6.3 \times 10$ min washes in deionized water

7. 1 each $15 \mathrm{~min}$ washes of $20 \%, 50 \%, 70 \%$, and $90 \%$ acetone in deionized water

$8.3 \times 20 \mathrm{~min}$ washes in $100 \%$ acetone

1:2 Epon/Spurr's [14] : acetone infiltration for $1 \mathrm{hr}$

Epon/Spurr's : acetone infiltration for $1 \mathrm{hr}$ temperature

13. $100 \%$ Epon/Spurr's resin

After the final fill with resin, mPrep/s capsules were inserted into an $\mathrm{mPrep} /$ bench as shown in Figure 1c, and, without dispensing the resin, the filled capsules were ejected from the pipettor. The $\mathrm{mPrep} /$ bench is a silicone 96-well plate that tightly fits the mPrep/s (and mPrep/g) capsule bottoms to keep fluid retained in the capsules. The mPrep/f couplers were then eved, and additional resin was added using a transfer with resin-filled capsules was then transferred to a $60^{\circ} \mathrm{C}$ oven for $\sim 12 \mathrm{hr}$ in-capsule polymerization. The Dieffenbachia plant tissue was prepared similarly except that $2 \%$ aqueous $\mathrm{OsO}_{4}$ was used for secondary fixation, and the resin was Epon $812(50 \%$ Epon 812, 20\% DDSA, 20\%NMA/ MNA, 10\% DBP with 2.5\% DMP-10 accelerator).

Sectioning. The mPrep/s capsules with polymerized epoxy blocks inside were directly clamped in the microtome chuck of a Leica U7 ultramicrotome. The block was trimmed and faced for microtomy in the standard way after first trimming away the end of the mPrep/s capsule (Figure 1d). Thick and thin sections were then cut using a diamond knife.

For light microscopy, $0.5 \mu \mathrm{m}$ kidney sections were cut and collected on glass slides, stained with polychrome I (methylene blue, azure II, 10\% methanol, 10\% glycerol), and examined with an Olympus $\mathrm{BH} 2$ microscope equipped with a Nikon 700 DSLR camera. For TEM, $70 \mathrm{~nm}$ sections were collected on 200-mesh Cu grids. One or two grids were then inserted and stored in labeled $\mathrm{mPrep} / \mathrm{g}$ capsules (Figure $2 \mathrm{a}$ ), and the capsules in turn were stored in an mPrep capsule grid box (not shown) until staining.

Grid staining and TEM examination. Grid-containing $\mathrm{mPrep} / \mathrm{g}$ capsules were connected via mPrep/f couplers to a single-channel pipettor (Figure 2b) or to an 8-channel Gilson Neo P8X200N Pipetman (Figure 2c). 

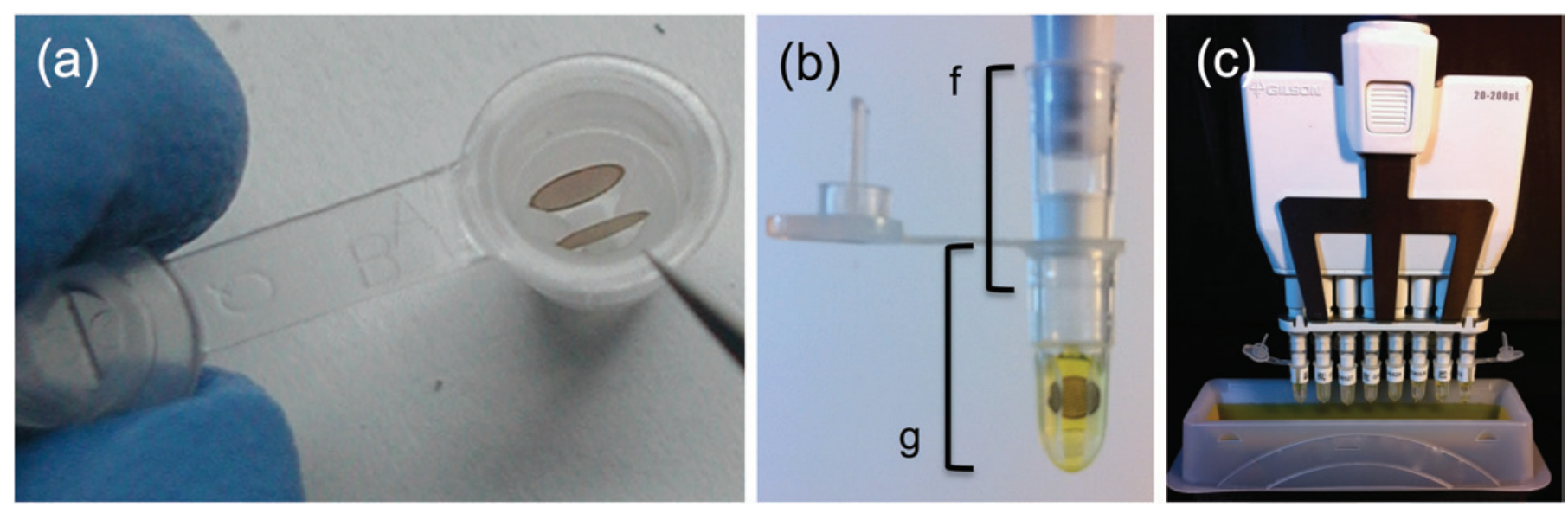

Figure 2: TEM grid staining using mPrep/g capsules: (a) mPrep/g capsule with two copper grids inserted. Forceps tips are visible to right. (b) mPrep/g capsule with grids filled with uranyl acetate stain [g] attached to a single-channel pipettor via an mPrep/f coupler [f]. (c) Eight labeled mPrep/g capsules containing a total of 16 grids attached to an 8-channel Pipetman via $\mathrm{mPrep} / \mathrm{f}$ couplers. Capsules are above a reagent reservoir filled with uranyl acetate stain.

With the 8-channel pipettor, 16 grids were simultaneously stained by aspirating $35 \mu \mathrm{l}$ of reagent into each $\mathrm{mPrep} / \mathrm{g}$ capsule from a reagent reservoir (Figure 2c), following this protocol:

\section{1. $2.5 \%$ (wt/vol) uranyl acetate in $50 \%$ ethanol for $14 \mathrm{~min}$ in the dark}

Table 1: Process and reagent efficiency to fix and embed 12 specimens using $\mathrm{mPrep} / \mathrm{s}$ and vial processing.

\begin{tabular}{|c|c|c|c|c|}
\hline \multirow[b]{2}{*}{ Process or Operation } & \multicolumn{2}{|c|}{$\begin{array}{c}\text { Manual } \\
\text { Operations }\end{array}$} & \multicolumn{2}{|c|}{$\begin{array}{c}\text { Reagent } \\
\text { Consumed (ml) }\end{array}$} \\
\hline & mPrep & Vial & mPrep & Vial \\
\hline $\begin{array}{l}\text { Place specimens into } \\
\mathrm{mPrep} / \mathrm{s} \text { or processing } \\
\text { vials }\end{array}$ & 12 & 12 & - & - \\
\hline Karnovsky's fix 1x & 1 & 12 & 1.2 & 12.0 \\
\hline Sorenson's buffer $3 x$ & 3 & 36 & 3.6 & 36.0 \\
\hline $\begin{array}{l}\text { Osmium tetroxide } \\
\text { buffer solution } 1 \times\end{array}$ & 1 & 12 & 1.2 & 12.0 \\
\hline Deionized water $3 x$ & 3 & 36 & 3.6 & 36.0 \\
\hline Uranyl acetate $1 \times$ & 1 & 12 & 1.2 & 12.0 \\
\hline Deionized water $3 x$ & 3 & 36 & 3.6 & 36.0 \\
\hline $\begin{array}{l}\text { Graded acetones in } \\
\text { water } 4 \times\end{array}$ & 4 & 48 & 4.8 & 48.0 \\
\hline $100 \%$ acetone $3 x$ & 3 & 36 & 3.6 & 36.0 \\
\hline Graded epoxies $3 x$ & 3 & 36 & 3.6 & 36.0 \\
\hline $100 \%$ epoxy $2 x$ & 2 & 24 & 2.4 & 24.0 \\
\hline $\begin{array}{l}\text { Transfer specimens } \\
\text { into embedding molds }\end{array}$ & 1 & 12 & 0 & 12.0 \\
\hline $\begin{array}{l}\text { Additional resin into } \\
\text { mold and insert label }\end{array}$ & 12 & 12 & 1.2 & 12.0 \\
\hline $\begin{array}{l}\text { Total User Operations } \\
\text { and Reagent } \\
\text { Consumed }\end{array}$ & 49 & 324 & 30.0 & 312.0 \\
\hline
\end{tabular}

"mPrep includes optional "topping off" of capsules with additional resin and optional insertion of a label into resin prior to curing. Vial assumes $2 \mathrm{ml}$ resin per embedding in flat or BEEM-type molds.
2. Deionized water rinses: 24 rapid aspiration and dispense rinses ( $\sim 2$ seconds/cycle) were done with fresh water, with the water replaced after every 8 rinses.

3. Reynold's lead citrate [11] for $9 \mathrm{~min}$. Note that because grids were inside mPrep capsules, no hydroxide $(\mathrm{NaOH})$ pellets were used, as commonly practiced with droplet staining to reduce carbon dioxide exposure that can lead to lead precipitates [12].

4. Rinses with deionized water as above

5. The $\mathrm{mPrep} / \mathrm{g}$ capsules were removed from the pipettor after dispensing the final rinse water.

6. Most grids were air dried in the $\mathrm{mPrep} / \mathrm{g}$ capsule. When faster drying was required for immediate TEM imaging, extraneous water was wicked from grids by inserting a wedge of filter paper into the capsule and touching the paper where the grids contact the inside of the capsule.

Grids were examined at $80 \mathrm{keV}$ in a Philips CM120 TEM and an FEI Tecnai 12 for rat and pig tissues. A JEOL 1200EX

Table 2: Process efficiency to stain 16 grids using mPrep/g processing and droplet staining.

\begin{tabular}{|l|c|c|}
\hline Operation & $\begin{array}{c}\text { mPrep/g capsule } \\
\text { operations }\end{array}$ & $\begin{array}{c}\text { Droplet } \\
\text { operations }\end{array}$ \\
\hline $\begin{array}{l}\text { Place grids in capsule } \\
\text { or grid box }\end{array}$ & 16 & 16 \\
\hline Uranyl acetate stain & 1 & 16 \\
\hline Water rinses & 1 & 16 \\
\hline $\mathrm{NaOH}$ pellets setup & $\begin{array}{c}\text { Not necessary, } \\
\text { sealed capsule }\end{array}$ & 1 \\
\hline Lead citrate stain & 1 & 16 \\
\hline Water rinses & 1 & 16 \\
\hline Wick off excess water & $16^{\star}$ & 16 \\
\hline Store grids in box & $\begin{array}{c}\text { Already in } \\
\text { storage capsule }\end{array}$ & 16 \\
\hline Total User Operations & $\mathbf{2 0}$ or $\mathbf{3 6}$ & $\mathbf{1 1 3}$ \\
\hline
\end{tabular}

"Wicking off excess water is optional with $\mathrm{mPrep} / \mathrm{g}$ processing, hence the range in total user operations. 

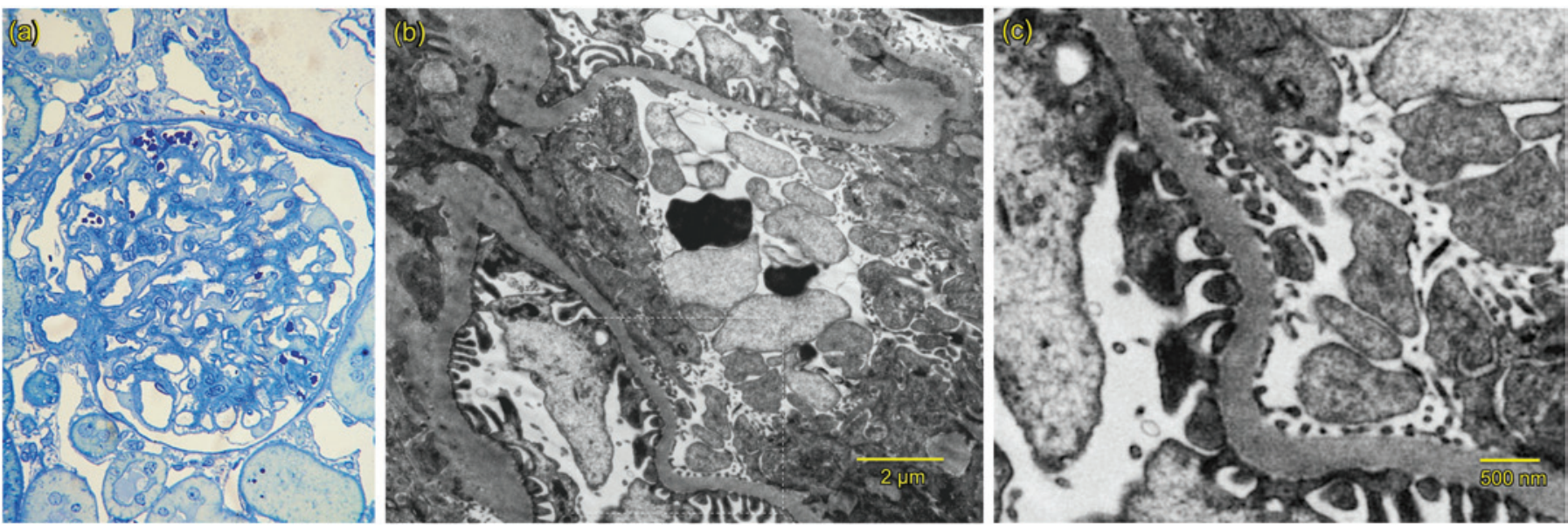

Figure 3: Rat (Rattus norvegicus) kidney tissue prepared using $\mathrm{mPrep} / \mathrm{s}$ and $\mathrm{mPrep} / \mathrm{g}$ capsules. (a) Rat kidney section stained with polychrome I, transmitted light microscopy. Image width about $100 \mu \mathrm{m}$. (b) Rat kidney TEM image from the same block as the light micrograph. The box indicates the region shown in Figure 3c. (c) Rat kidney TEM image at higher magnification.
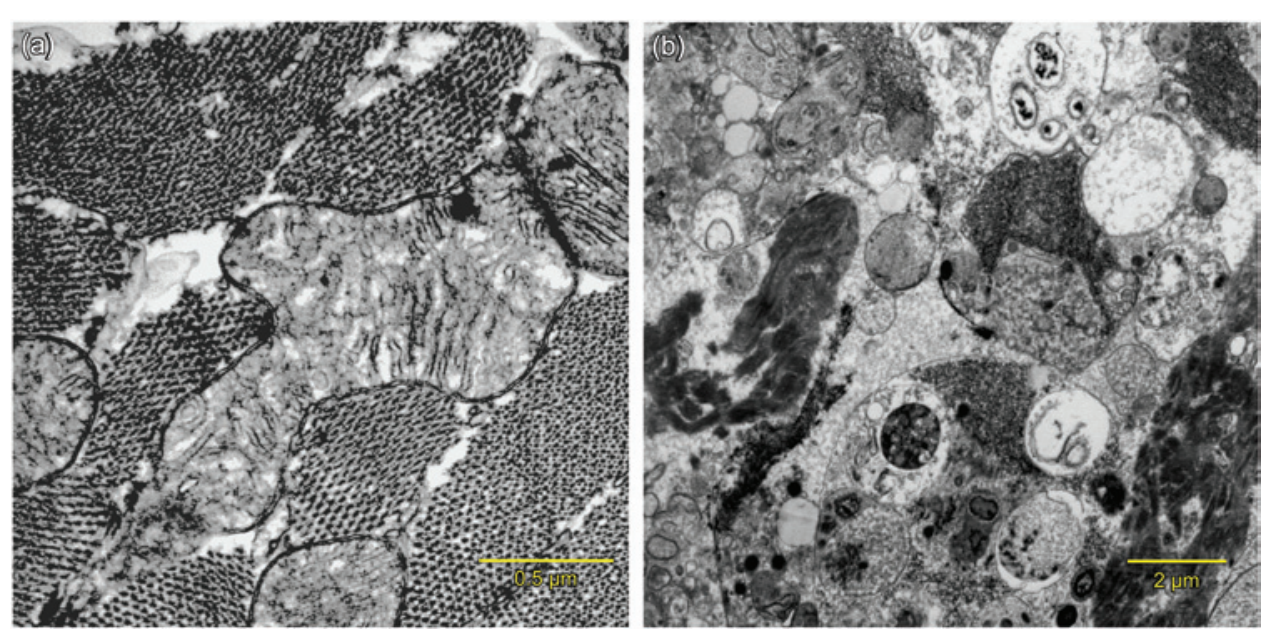

Figure 4: Porcine tissues entirely prepared using mPrep/s and mPrep/g capsules. (a) Porcine heart TEM showing myofibrils in various orientations and mitochondria. (b) Porcine skin TEM. micrographs indicate good tissue fixation and uniform staining. Figure 5 shows vampire bat brain sections stained using mPrep/g processing that exhibit uniform staining without precipitates. Figure 6 shows Dieffenbachia prepared using mPrep/s and $\mathrm{mPrep} / \mathrm{g}$ capsule processing by technical college students learning electron microcopy. This specimen also shows good fixation and uniform staining.

Labor efficiency and reagent consumption. The method for processing tissue with $\mathrm{mPrep} / \mathrm{s}$ capsules was described in the Materials and Methods section of this article. The most commonly repeated steps were the 24 fluid reagent exchanges from was used for the Dieffenbachia specimen and a Hitachi H-7600 for the vampire bat brain.

Measuring reagent consumption and labor efficiency. Reagent consumption for mPrep processing was compared to common vial and transfer pipette methods. The reagent consumed for processing 12 tissue specimens with $\mathrm{mPrep} / \mathrm{s}$ capsules was measured with the pipettor. Reagent that would have been used in standard vial processing was calculated assuming $1 \mathrm{ml}$ per reagent exchange. The reagent consumed for grid processing was not compared because mPrep/g capsules use $17.5 \mu$ l per grid (two grids in one $35 \mu \mathrm{l}$ capsule), which is similar to typical droplet volumes of $25-100 \mu l$ per grid. The labor efficiency for processing 12 specimens and 16 grids was calculated by counting the number of physical operations required for mPrep capsule processing and the number of physical operations that would be used for vial tissue processing and droplet grid staining, as enumerated in Tables 1 and 2.

\section{Results}

Images of processed tissues. Three mammalian tissues and resultant sections on grids were entirely prepared using mPrep capsule processing. Figure 3 shows rat kidney imaged with LM and TEM, and Figure 4 shows pig heart and skin tissue. These fixative to final resin (Table 1). As shown in Figure 1, all these reagent exchanges were performed simultaneously by immersing the bottom ends of $12 \mathrm{mPrep} / \mathrm{s}$ capsules into reagent-containing reservoirs and operating the pipettor. Final embedding was achieved by simultaneously inserting all 12 resin-filled capsules into the silicone bench holder prior to transfer into the curing oven. Compared to vial processing, the number of manual operations was reduced 7 -fold, from 324 to 49 operations with $\mathrm{mPrep}$ capsule processing. The total reagent consumed in preparing 12 specimens with $\mathrm{mPrep} / \mathrm{s}$ capsules was $30 \mathrm{ml}$, compared to $312 \mathrm{ml}$ calculated for vial processing, providing a 10 -fold reduction (Table 1). Table 2 shows that simultaneous staining of 16 grids with $\mathrm{mPrep} / \mathrm{g}$ capsules required as few as 20 operations, compared to over 100 calculated for droplet staining.

\section{Discussion}

Conventional processing methods are well established. Thus, the mPrep capsule methodology introduced here will be discussed in relation to these known methods.

Quality. As shown in Figures 3-6, with mPrep capsule processing all specimens were well preserved and stained cleanly 


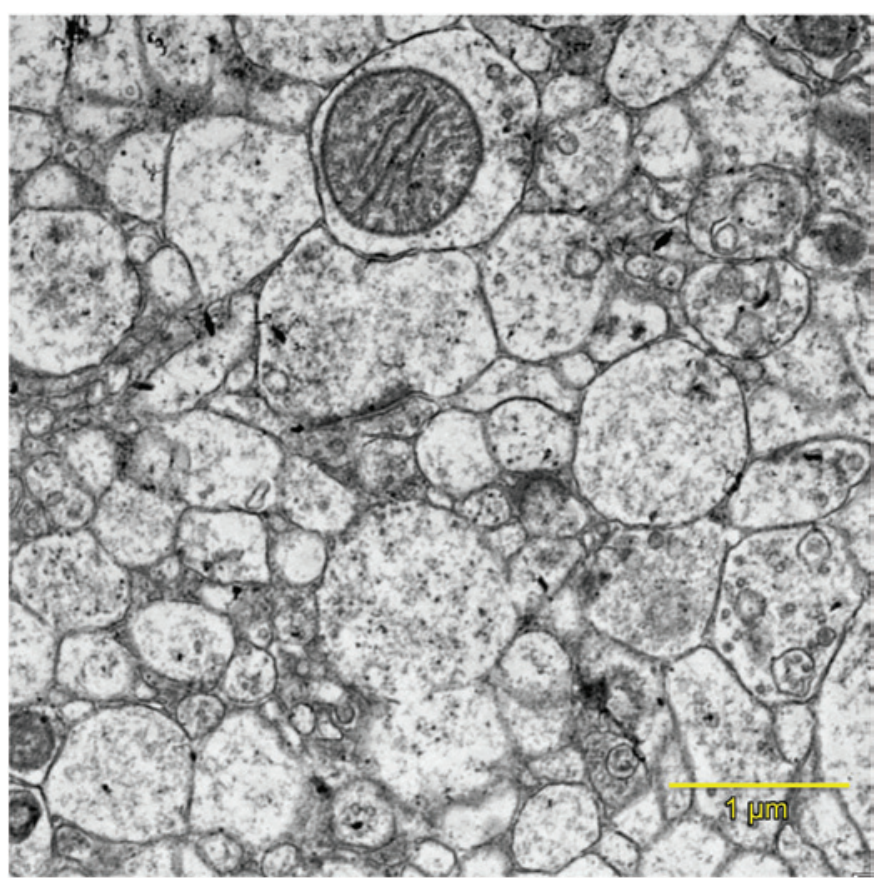

Figure 5: Vampire bat (Desmondontidae) brain prepared using $\mathrm{mPrep} / \mathrm{g}$ grid staining.

and intensely. This is expected because the chemistry of sample preparation and staining were unchanged; only the devices used for delivering reagents and the reagent volumes were different. With mPrep capsule processing there is also the ability to improve quality and experimental reproducibility. This is because mPrep capsule pipetting provides simultaneous reagent delivery to multiple specimens thus providing identical reagent reaction times for all specimens. Secondly, because reagent exchanges occur very rapidly ( $\leq 1$ second), the potential for accidental air drying that can occur with vial processing is nearly eliminated. Thirdly, because specimens are safely entrapped, the potential for damage caused by the specimen sticking to the side of vials, floating on the reagent surface, or being accidently sucked into transfer pipettes, is eliminated. Similarly, because grid handling is nearly eliminated, the probability for grid damage or loss is reduced, while simultaneous staining improves reproducibility.

Reagent efficiency. Because $\mathrm{mPrep} / \mathrm{s}$ capsule volume is sized for TEM specimens, specimen processing only required $30 \mathrm{ml}$ for 12 specimens $(2.5 \mathrm{ml}$ per specimen), as compared to a calculated reagent consumption of $26 \mathrm{ml}$ per specimen for vial processing based on using only $1 \mathrm{ml}$ per each vial reagent exchange (Table 1). This 10 -fold reduction is substantial, yet it could be further reduced because as little as $10 \mu 1$ per reagent exchange can be used with small specimens entrapped at the bottom of the capsules. Thus, the potential to reduce consumption by about 100 -fold exists, which is especially desirable with expensive reagents such as antibodies, gold labels, or toxic compounds.

Operational efficiency. Simultaneous processing with multi-channel pipetting not only can improve reproducibility, but also can greatly reduce labor operations (Tables 1 and 2).

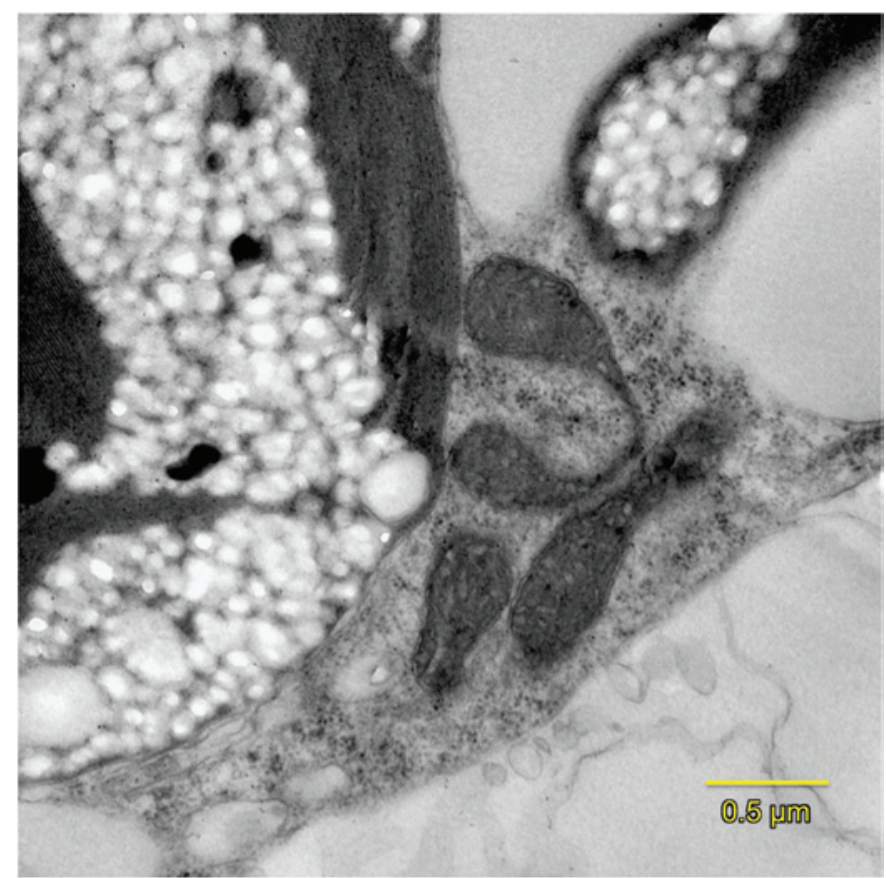

Figure 6: Dieffenbachia (dumb cane) prepared using mPrep/s and mPrep/g capsules.

Note that the simple counting of operations in Tables 1-2 does not fully characterize the improved operational ease. For mPrep reagent exchanges, minimal attention is required because specimens and grids are safely encapsulated, and only a simple depression of the pipettor aspirate-dispense button is required. With $\mathrm{mPrep} / \mathrm{s}$ capsules, specimens are only directly handled when inserted into the capsule and when the block was prepared for sectioning. Sectioning preparation is also easier because $\mathrm{mPrep} / \mathrm{s}$ capsules are directly chucked into the microtome and faced through the capsule (Figure 1d). With respect to grid processing, grids are only handled when they are inserted into an mPrep/g capsule and when removed from the microtome to insert into the TEM stage, thus greatly reducing operator attention in comparison to most staining methods. An additional effort reduction is that Prep/g capsules reduce air exposure so that lead citrate staining does not require hydroxide pellets to sequester carbon dioxide [12].

Enabling multiple protocol processing using microtiter plates. In the present study, specimens and grids were processed using multi-channel pipettes with reagent drawn from a single reagent reservoir (Figures $1 \mathrm{~b}$ and $2 \mathrm{c}$ ), thus all specimens and grids were processed with the same reagents. However, by using the innovation of the 96-well microtiter plate [7-9], it is just as easy to deliver different reagents to each of the mPrep capsules. This is simply achieved by placing different reagents, titrations, or stains into individual microtiter wells, and by using an 8- or 12-channel pipettor to draw reagents into mPrep capsules from different wells. This is shown in the Application Notes section of the Microscopy Innovations website where a template can guide protocols such as immuno-labeling [15]. This template methodology can be extended to many specimen or grid applications, such as the study where McClain simultaneously 
evaluated 16 different tissue fixation, embedding, and staining protocols [16].

\section{Conclusion}

The mPrep capsule processing method can improve the ease, efficiency, and reproducibility of TEM and SEM sample preparation protocols. Capsule processing can provide advantages that include identical and simultaneous preparation to increase reproducibility, to provide ten-fold or greater reduction of reagent consumption, and to afford a similar reduction in technician effort. This report shows that efficient processing, producing quality results, was achieved by multiple labs in three different institutions.

\section{Acknowledgments}

The authors acknowledge the assistance of Patricia Kierski and Jonathon McAnulty (University of Wisconsin School of Veterinary Medicine) for providing fresh pig tissues, and Janice Pennington (Department of Botany, University of Wisconsin) for helpful advice. We also acknowledge the use of instrumentation supported by the University of Wisconsin Advanced Materials Industrial Consortium (DMR-1121288) and the Nanoscale Science and Engineering Center (DMR-0832760).

\section{References}

[1] GE Palade, J Exptl Med 95 (1952) 285-98.

[2] KR Porter and J Blum, Anat Rec 117 (1953) 685-710.
[3] A Claude and EF Fullam, J Exp Med 83 (1946) 499-504.

[4] K Porter et al., J Exp Med 81 (1945) 223-46.

[5] GE Palade, Science 189 (1975) 347-58.

[6] WE Gilson and RE Gilson, Adjustable Pipette. US Patent 3827305A, filed Oct 24, 1972, published Aug 6, 1974.

[7] NM Cooke and PH Hall, US Patent 3356462A, filed Aug 9, 1966, published Dec 5, 1967.

[8] R Manns. Microplate History, 2nd Ed., presented at MipTec-ICAR'99, May 17-21, 1999, Montreux, Switzerland.

[9] T Astle, Journal of the Association for Laboratory Automation 5 (2000) 30-31.

[10] JJ Bozzola and LD Russell, Electron Microscopy, 2nd Ed., Jones and Bartlett, Boston, 1999, p. 19.

[11] ES Reynolds, J Cell Biol 17(1) (1963) 208-12.

[12] JJ Bozzola and LD Russell, Electron Microscopy, 2nd Ed., Jones and Bartlett, Boston, 1999, p. 126-29.

[13] MJ Karnovsky, J Cell Biol 27 (1965) 137A.

[14] KD Wendt et al., J Microsc 214 (Pt 1) (2004) 80-88.

[15] Microscopy Innovations, LLC, "Immunolabeling with mPrep Capsules - Protocol, and Template for Laying Out Reagents in 96-Well Plates," in "Application Notes." http://microscopyinnovations.com/Tech-info.php (accessed July 3, 2015).

[16] M McClain, Microsc Microanal 20 (Suppl 3) (2014) 1288-89.

\section{Evactron ${ }^{\circledR}$ De-Contaminators FAST, Large Volume, and Damage Free Plasma Cleaning}

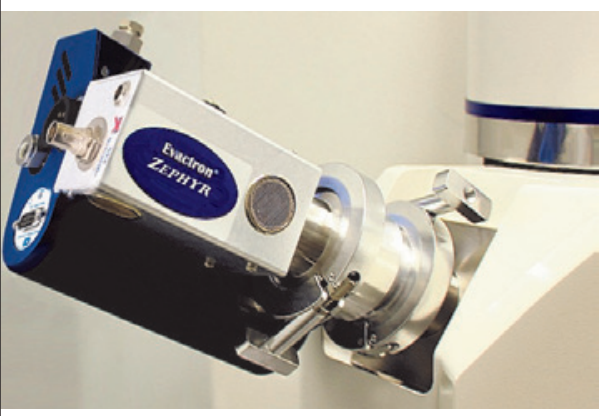

Evactron $\mathbb{R}^{\text {Zephyr }}{ }^{\text {TM }}$ Remote Plasma Cleaner with Advanced Technology
Evactron $^{\circledR}$

By XEI Scientific

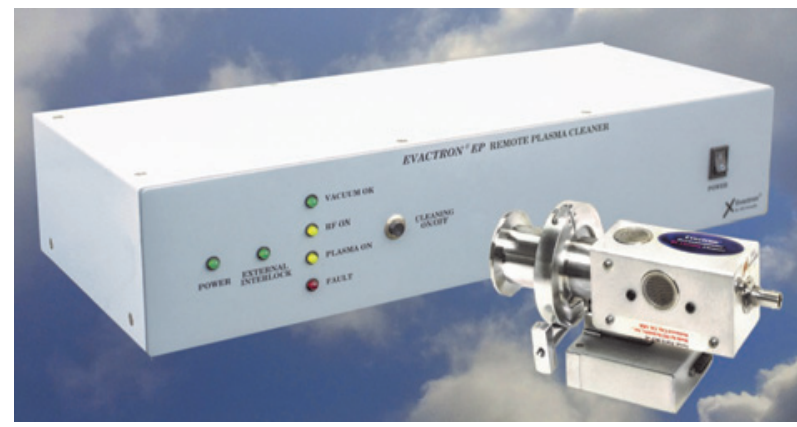

Evactron ${ }^{\circledR}$ EP D-C

Same High Performance at a more Affordable Price 


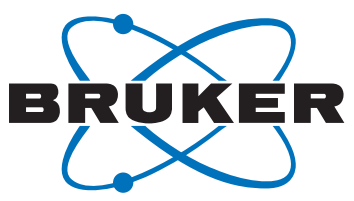

\section{Techniques - 1 Workflow.}

ESPRIT 2, the

only software

which combines

4 microanalysis methods.
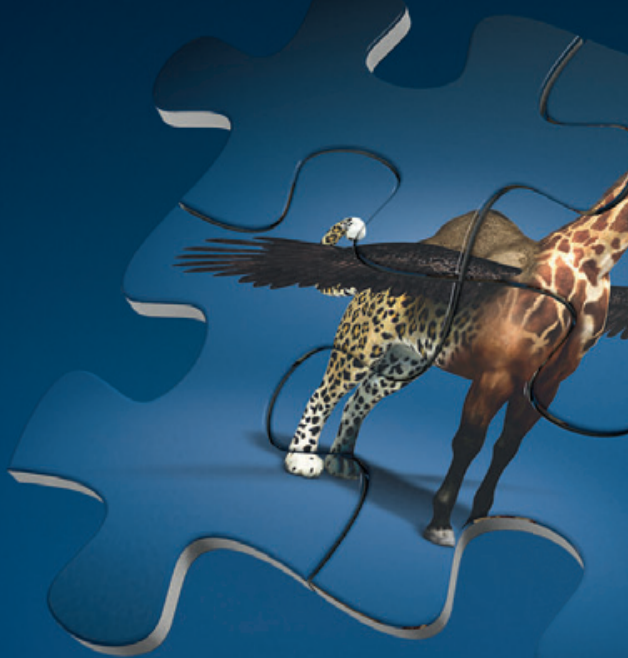

(1)
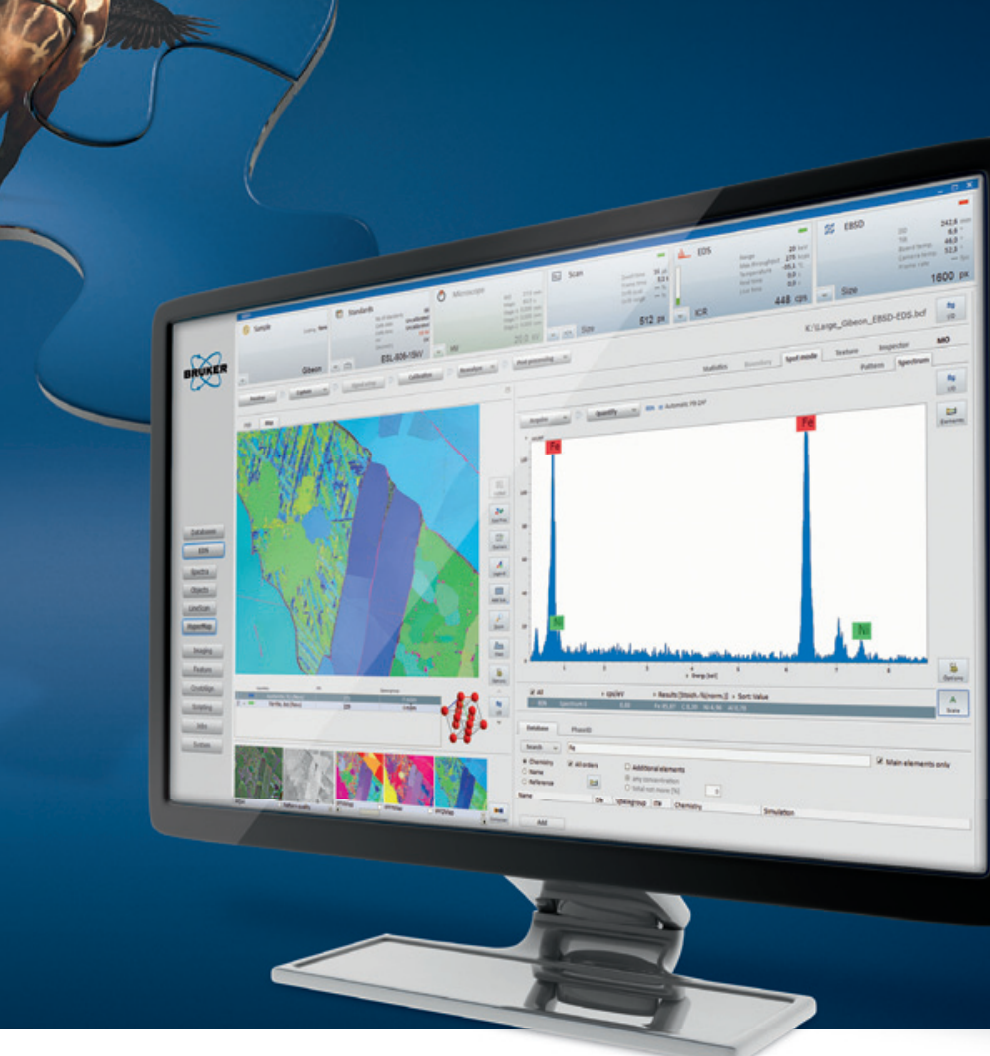

- Comprehensive management of analysis and results from EDS, WDS, EBSD and Micro-XRF with one software

- Complementary techniques provide you the most accurate and reliable results

- Zeta factor quantification for characterization of thin layers

Someone has to be first.

\section{$\square$ www.bruker.com/esprit2}

1.

wirit

口ath

Innovation with Integrity 